\title{
SINGULARITIES OF ABEL-JACOBI MAPS AND GEOMETRY OF DISSOLVING VORTICES
}

\author{
NUNO M. ROMÃO
}

\begin{abstract}
Gauged vortices are configurations of fields for certain gauge theories in fibre bundles over a surface $\Sigma$. Their moduli spaces support natural $L^{2}$-metrics, which are Kähler, and whose geodesic flow approximates vortex scattering at low speed. This paper focuses on vortices in line bundles, for which the moduli spaces are modeled on the spaces $\Sigma^{(k)}$ of effective divisors on $\Sigma$ with a fixed degree $k$; we describe the behaviour of the underlying $L^{2}$-metrics in a "dissolving limit" where the $L^{2}$-geometry simplifies. In such limit, the metrics degenerate precisely at the singular locus of the Abel-Jacobi map AJ of $\Sigma$ at degree $k$, and their geometry can be understood in terms of the variety $W_{k}=\operatorname{AJ}\left(\Sigma^{(k)}\right)$ inside the Jacobian of $\Sigma$. Some intuition about the behaviour of the geodesic flow close to a singularity is provided through the study of the simplest example (resolution of a double point on a surface), corresponding to two dissolving vortices moving on a hyperelliptic curve of genus three.
\end{abstract}

\section{Introduction}

The vortex equations originate in the Ginzburg-Landau theory of superconductivity [4] and describe static, stable solutions of certain $(2+1)$-dimensional gauge theories [13, 9, 33, 23]. In the simplest example, the equations relate a connection $\mathrm{d}_{a}$ on a principal $\mathrm{U}(1)$-bundle over a smooth surface with Kähler structure $\left(\Sigma, j_{\Sigma}, \omega_{\Sigma}\right)$, which we will assume to be compact, and a section $\phi$ of an associated line bundle $\mathcal{L} \rightarrow \Sigma$. As part of the geometric setup, one fixes a Hermitian structure on this line bundle, which equips each fibre $\mathcal{L}_{P} \cong \mathbb{C}$ with a symplectic structure preserved by the $\mathrm{U}(1)$-action. This action is Hamiltonian, and a moment map $\mu: \mathcal{L} \rightarrow \mathfrak{u}(1)^{*} \cong \mathbb{R}$ is specified globally as

$$
\mu(w)=\frac{1}{2}(\langle w, w\rangle-\tau), \quad \text { for } \quad w \in \mathcal{L}_{P}, \quad P \in \Sigma
$$

where $\tau \in \mathbb{R}$ is a constant (which remains arbitrary a priori). In this setup, the vortex equations read

$$
\begin{gathered}
\bar{\partial}_{a} \phi=0, \\
F_{a}+(\mu \circ \phi) \omega_{\Sigma}=0 .
\end{gathered}
$$

The first equation expresses that the section $\phi: \Sigma \rightarrow \mathcal{L}$ is holomorphic, i.e. annihilated by the operator $\bar{\partial}_{a}: \Omega^{0}(\Sigma, \mathcal{L}) \rightarrow \Omega^{1}(\Sigma, \mathcal{L})$ defined from the unitary connection $\mathrm{d}_{a}$ and the complex structure on $\Sigma$ [8], while the second equation relates the curvature $F_{a}=\mathrm{d} a$ of the connection to the moment map evaluated on the values of the section and the area form $\omega_{\Sigma}$.

By integrating (1.3) over $\Sigma$, one finds that the squared $L^{2}$-norm $\|\phi\|_{L^{2}}^{2}:=\int_{\Sigma}\langle\phi, \phi\rangle \omega_{\Sigma}$ satisfies

$$
\|\phi\|_{L^{2}}^{2}=\tau V-4 \pi k
$$

The author is thankful to the organisers of the Workshop on Singularities in Geometry and Applications for the opportunity to present this work, and for hospitality at the Mathematical Research and Conference Center in Będlewo, Poland. 
where $V:=\int_{\Sigma} \omega_{\Sigma}$ is the total area of the surface and $k=\frac{1}{2 \pi} \int_{\Sigma} F_{a}$ is the first Chern class (or degree) of the line bundle. Sometimes, $k$ is referred to as the vortex number. Since the squared $L^{2}$-norm is nonnegative, (1.4) implies that a necesssary condition for solutions of the vortex equations to exist is $\tau \geq \frac{4 \pi k}{V}$. A theorem of Bradlow [6] (see also [10]) asserts that, if we take $\tau>\frac{4 \pi k}{V}$, one can find for any effective divisor $D$ of degree $k$ on $\Sigma$ a unique solution of the equations up to gauge equivalence which satisfies $(\phi)=D$; this is what is called a $k$-vortex. The moduli space $\mathcal{M}_{k}$ of $k$-vortices is therefore the symmetric product $\Sigma^{(k)}=\Sigma^{k} / \mathfrak{S}_{k}$, a smooth complex manifold with complex dimension $k$.

The divisor of zeroes $(\phi)$ is the most basic object one can assign to a $k$-vortex and it gives the precise location of $k$ individual vortex cores, but these objects should be thought of as extending over $\Sigma$ and interacting with each other. Interesting information about vortex interactions is encoded in a natural metric on the moduli space, which is induced from the trivial $L^{2}$-metric on the space of all fields $\left(\mathrm{d}_{a}, \phi\right)$ by an infinite-dimensional analogue of symplectic reduction. The induced metric is nontrivial and also Kähler with respect to the complex structure on $\Sigma^{(k)}$ induced from $j_{\Sigma}$. We use the term ' $L^{2}$-geometry' to refer to this family of Kähler structures on each $\mathcal{M}_{k}$, which is parametrised by $\left.\tau \in\right] \frac{4 \pi k}{V}, \infty[$.

To be more precise, the $L^{2}$-metrics are defined at each $k$-vortex solution $\left(\mathrm{d}_{a}, \phi\right)$ by

$$
\|(\dot{a}, \dot{\phi})\|_{\left(\mathrm{d}_{a}, \phi\right), L^{2}}^{2}=\int_{\Sigma}\left(\frac{1}{2} \dot{a} \wedge \star \dot{a}+\langle\dot{\phi}, \dot{\phi}\rangle \omega_{\Sigma}\right)
$$

where $\dot{a} \in \Omega^{1}(\Sigma)$ and $\dot{\phi} \in \Gamma(\Sigma, \mathcal{L})$ are fields representing tangent vectors in $\mathrm{T}_{\left(\mathrm{d}_{a}, \phi\right)} \mathcal{M}_{k}$ (they satisfy the linearisation of the vortex equations about $\left(\mathrm{d}_{a}, \phi\right)$ and are $L^{2}$-orthogonal to the orbit of the gauge group through this point), and $\star$ is the Hodge star of the Kähler metric on $\Sigma$. Integrals over $\Sigma$ such as (1.5) would seem hopeless to compute directly, but it turns out that they localise onto the support of the divisor $(\phi)$ associated to the vortex [30, 29]. This feature has been invaluable to understand the $L^{2}$-metrics and their physical content. Even though an explicit calculation of the metrics seems to be beyond reach as yet, some results have been obtained in certain regimes, adding to our intuition about the geometry underlying the vortex equations. For example, formulas for the symplectic volume of the moduli spaces $\mathcal{M}_{k}$ have been established by Manton and Nasir [21] exploring localisation:

$$
\operatorname{Vol}\left(\mathcal{M}_{k}\right)=(2 \pi)^{2 k} \sum_{n=0}^{\min \{k, g\}} \frac{g !}{n !(k-n) !(g-n) !}\left(\frac{\tau V}{4 \pi}-k\right)^{k-n} .
$$

The $L^{2}$-metrics encode precious information about infinite-dimensional dynamical models that incorporate solitons. For instance, their geodesic flow is of direct physical interest, since it gives a good approximation to the slow dynamics in the Abelian Higgs model in $2+1$ dimensions [23, 31].

One regime in which the $L^{2}$-geometry becomes somewhat tractable is what we call the dissolving limit, which corresponds to taking

$$
\tau \rightarrow \frac{4 \pi k}{V}
$$

for $\Sigma$ compact. This was considered in [32] and [3] when $\Sigma$ has genus $g=0$; and for genus $g \geq 1$ first by Nasir [26], and then by Manton and Romão [22]. In the following, we shall give an account of the results in [22] — we will add little to the presentation in the original paper, and shall focus on the $k>1$ (multivortex) case, which brings in some interesting issues that relate to the theory of singularities. 


\section{VORTICES IN THE DISSOLVING LIMIT}

We would like to understand the geometry of the moduli space of vortices in the dissolving limit (1.7), which will turn out to be a simplification of the full $L^{2}$-geometry. It is instructive to look first at the space of solutions of the equations when one sets $\tau=\frac{4 \pi k}{V}$. Then equation (1.4) implies that $\phi=0$, so the first vortex equation (1.2) is trivially satisfied: the zero section is always holomorphic. Note that the action of $\star$ on $\Omega^{1}(\Sigma)$ depends on $j_{\Sigma}$ alone, so when $\phi \rightarrow 0$ we expect that the $L^{2}$-metric, defined by the expression (1.5), will only depend on the conformal class of the metric given on $\Sigma$. In the following, we shall make this observation more precise.

The second vortex equation (1.3) simplifies to

$$
F_{a}=\mathrm{d} a=\frac{\tau}{2} \omega_{\Sigma}
$$

which says that the curvature of the connection $\mathrm{d}_{a}$ is a constant multiple of the area form $\omega_{\Sigma}$. Notice that the constant of proportionality $\frac{\tau}{2}=\frac{2 \pi k}{V}$ is determined by the topology and the normalisation $V$. This is still a crude approximation to the degeneration of the moduli space of $k$-vortices that we are interested in; we introduce the following terminology:

Definition 2.1. A dissolved $k$-vortex is a solution $\mathrm{d}_{a}$ to equation $(2.1)$ in a line bundle of degree $k$.

Dissolved vortices correspond to "constant curvature" or "projectively flat" connections with respect to the 2 -form $\omega_{\Sigma}$, and they are parametrised by the dual to the Jacobian variety of $\Sigma$, a complex $g$-torus if $\Sigma$ has genus $g$. Recall that the Jacobian is defined by [11]

$$
\operatorname{Jac}(\Sigma)=H^{0}\left(\Sigma, K_{\Sigma}\right)^{*} / H_{1}(\Sigma, \mathbb{Z}) .
$$

Here, $K_{\Sigma}$ denotes the (canonical) sheaf of holomorphic 1 -forms, and the inclusion $H_{1}(\Sigma, \mathbb{Z}) \hookrightarrow$ $H^{0}\left(\Sigma, K_{\Sigma}\right)^{*}$ is provided by integration over 1-cycles: $\lambda \mapsto \oint_{\lambda}$. If we are given a solution $\mathrm{d}_{a}$ (in a unitary trivialisation, $\mathrm{d}_{a}=\mathrm{d}-\mathrm{i} a$ for a real 1-form $a$ ) of equation (2.1), for example constructed out of local symplectic potentials of $\omega_{\Sigma}$ obtained from Kähler potentials, we can write any other solution modulo gauge transformations as $\mathrm{d}_{a+\alpha}$ where $\alpha$ is a global harmonic 1-form (in other words, through twisting by a flat line bundle with connection $\left.\mathrm{d}_{\alpha}\right) ; \alpha$ satisfies

$$
\mathrm{d} \alpha=0 \quad \text { and } \quad \mathrm{d} \star \alpha=0 .
$$

The first equation in (2.3) follows from (2.1), while the second equation provides a section from the space of gauge orbits.

Different dissolved vortices have the same curvature 2-form but different holonomies around 1-cycles in $\Sigma$. In fact, one should identify dissolved vortices if they have the same holonomies, and this corresponds to quotienting the real $2 g$-dimensional vector space of harmonic 1-forms $\alpha$ by the lattice of rank $2 g$ defined by the relations

$$
\oint_{\lambda} \alpha \in 2 \pi \mathbb{Z}, \quad \forall \lambda \in H_{1}(\Sigma, \mathbb{Z}),
$$

thus we end up with the dual torus to $\operatorname{Jac}(\Sigma)$, as claimed. For some purposes, it is useful to think of a harmonic 1-form as the real part of a holomorphic 1-form on $\Sigma$, and so there is also a complex structure involved (more explicitly, « plays the role of complex structure at each point of the torus). Thus we are really dealing with the geometry of Abelian varieties [11].

Now the dual space $H^{0}\left(\Sigma, K_{\Sigma}\right)^{*}$ has a canonical inner product, namely the polarisation of the Jacobian [11]. One can think of it as the flat Kähler metric associated to the natural complex structure induced by $j_{\Sigma}$, together with the symplectic form obtained by extending the intersection pairing on $H_{1}(\Sigma, \mathbb{Z})$ to real coefficients (note that $H_{1}(\Sigma, \mathbb{Z}) \otimes_{\mathbb{Z}} \mathbb{R}=H^{0}\left(\Sigma, K_{\Sigma}\right)^{*}$, 
cf. (2.2)); of course, this structure is invariant under translations. We shall denote by $\Omega_{J}$ the $(1,1)$-form of this Kähler metric on $\operatorname{Jac}(\Sigma)$. The map

$$
\omega \mapsto \frac{1}{2 \pi} \int \alpha \wedge \omega
$$

provides an isomorphism relating infinitesimal flat connections $\alpha$ and elements of the dual space $H^{0}\left(\Sigma, K_{\Sigma}\right)^{*}$, and therefore a pull-back of the polarisation to a Kähler structure on the dual torus to $\operatorname{Jac}(\Sigma)$, yielding a metric at each point

$$
(\alpha, \beta) \mapsto \int_{\Sigma} \alpha \wedge \star \beta
$$

It is not hard to see that this induced metric coincides with the natural $L^{2}$-geometry on the space of dissolved vortices (see Section 3 of [22] for the explicit argument). This geometry on the dual Jacobian is independent of the first Chern class $k$, the vortex number of the dissolved vortex.

To understand the $L^{2}$-geometry of $k$-vortices in the dissolving limit (1.7), a more insightful notion is the following.

Definition 2.2. A dissolving $k$-vortex is a unitary connection $\mathrm{d}_{a}$ on a line bundle of degree $k$ whose induced holomorphic structure $\bar{\partial}_{a}$ has nontrivial kernel.

In other words, for a dissolving vortex one requires the existence of a nonzero holomorphic section for the induced holomorphic structure. So to a dissolving vortex one can always associate a dissolved vortex, but not conversely, and we should be able to think of it as a limit $\left(\mathrm{d}_{\mathrm{a}}, 0\right)$ of a sequence of $k$-vortices as $\tau \rightarrow \frac{4 \pi k}{V}$.

Recall that the Jacobian variety $\operatorname{Jac}(\Sigma)$ plays another important role, namely that of classifying holomorphic line bundles over $\Sigma$ of a given degree [11]. Holomorphic line bundles are determined by divisor classes (i.e. divisors on $\Sigma$ modulo linear equivalence, where two divisors of the same degree are identified if their difference is the divisor of zeroes and poles of a global meromorphic function on $\Sigma$ ). The relation between divisors on $\Sigma$ and $\operatorname{Jac}(\Sigma)$ is achieved via the Abel-Jacobi map, which depends on the choice of a basepoint $P_{0} \in \Sigma$ : to a divisor $D=D_{+}-D_{-}$, where $D_{+}=\sum_{i} P_{i}$ and $D_{-}=\sum_{j} Q_{j}$ are effective divisors, $\operatorname{AJ}(D)$ is defined by a linear functional on holomorphic 1-forms via the Abelian integrals

$$
\operatorname{AJ}(D): \omega \mapsto \sum_{i} \int_{P_{0}}^{P_{i}} \omega-\sum_{j} \int_{P_{0}}^{Q_{j}} \omega .
$$

The value determined by this quantity in the Jacobian variety does not depend on the choice of paths connecting each $P_{i}$ or $Q_{j}$ to $P_{0}$ since the ambiguity lies on the image of $H_{1}(\Sigma, \mathbb{Z})$ in $H^{0}\left(\Sigma, K_{\Sigma}\right)^{*}$. Moreover, a different choice of basepoint $P_{0}$ simply leads to a translation in the Jacobian. We will be interested in the restriction of the Abel-Jacobi maps $\mathrm{AJ}_{k}$ to the spaces of effective divisors of degree $k>0$, which are the symmetric products $\Sigma^{(k)}$ and can be identified with moduli spaces of $k$-vortices.

Note that the maps $\mathrm{AJ}_{k}$ are holomorphic. Their images

$$
W_{k}:=\operatorname{AJ}_{k}\left(\Sigma^{(k)}\right) \subset \operatorname{Jac}(\Sigma)
$$

are complex subvarieties of dimension $\min \{k, g\}$, and they can be regarded as the spaces of dissolving $k$-vortices. It is a classical theorem of Abel [11] that the map

$$
\mathrm{AJ}_{1}: \Sigma^{(1)}=\Sigma \longrightarrow \operatorname{Jac}(\Sigma)
$$


is an embedding. So the flat Kähler structure associated to the polarisation of the Jacobian variety, discussed above, induces a Kähler structure $\mathrm{AJ}_{1}^{*} \Omega_{J}$ on $\Sigma$. In [22], the following result is proven:

Theorem 2.3. In the dissolving limit (1.7), the $L^{2}$-metric on $\mathcal{M}_{1}$ converges to a natural Bergman metric on $\Sigma$, regarded as the moduli space of one dissolving vortex. It coincides with the Kähler metric obtained by pulling back the polarisation of the Jacobian via the Abel-Jacobi embedding $\mathrm{AJ}_{1}: \Sigma \hookrightarrow \operatorname{Jac}(\Sigma)$.

The idea of the proof is to relate Hecke modifications performing shifts of the line bundle associated to a dissolving 1-vortex to complex gauge transformations; such a shift can also be described by addition of harmonic 1-forms at the level of the connections associated to the holomorphic structures, and their length for an infinitesimal shift describes the $L^{2}$-metric, which can be computed in holomorphic coordinates. For our purposes, a Bergman metric $[18,14]$ on a compact Riemann surface of genus $g \geq 1$ is a Riemannian structure of the form

$$
\mathrm{d} s^{2}=\sum_{j=1}^{g} \omega_{j} \bar{\omega}_{j},
$$

which is associated to any basis $\left\{\omega_{1}, \ldots, \omega_{g}\right\}$ of $H^{0}\left(\Sigma, K_{\Sigma}\right) \cong \mathbb{C}^{g}$; see Appendix A in [22]. Note that (2.8) is constant on $\mathrm{U}(g)$-orbits of the space of bases. The particular Bergman metric in our result is the one coming from an orthogonal basis with respect to the metric on 1-forms given by (2.6), up to the global factor of $(2 \pi)^{2}$.

\section{Geometry of Dissolving multivortices}

Important details about the complex geometry of the Riemann surface $\Sigma$ are captured by the dissolving limit of the $L^{2}$-geometry of the moduli spaces of vortices. We have argued that the limit Kähler structure should depend only on the intrinsic complex structure of $\Sigma$, and it would be interesting to understand how this dependence is reflected qualitatively in its curvature properties and the geodesic flow, for example. In the $k=1$ case, the Bergman metric on $\mathcal{M}_{1}=\Sigma$ in Theorem 2.3 is known to have nonpositive Gauß curvature [19]. This follows from general facts: the image of a holomorphic embedding $\Sigma \hookrightarrow \operatorname{Jac}(\Sigma)$ of a complex curve in a Kähler manifold must be a minimal surface, so its principal curvatures at each point must be symmetric. If $g>1$, it turns out that the curvature vanishes at most at a finite number of points, which are precisely the Weierstraß points [11] of $\Sigma$ if $X$ is hyperelliptic (otherwise the curvature never vanishes) [19]. For Kähler structures of dissolving multivortices, one should be able to obtain results in this spirit, but the geometry in higher dimensions will be richer.

In what follows, we shall explore the dissolving limit (1.7) for multivortices, assuming that the two inequalities

$$
1<k<g
$$

hold. We have already stated that the image (2.7) of the moduli space of vortices $\mathcal{M}_{k}=\Sigma^{(k)}$ under the Abel-Jacobi map (well defined once a base point $P_{0} \in \Sigma$ is chosen, and holomorphic) is a complex subvariety of the Jacobian. However, as we will make more precise in a moment, for large enough $k$ this map is no longer an embedding, in contrast to the $k=1$ case, and then the images $W_{k}$ in (2.7) are singular subvarieties. Among these objects, perhaps the most familiar one is $W_{g-1}$, which is a translation of the $\Theta$-divisor [11] in $\operatorname{Jac}(\Sigma)$ and has singularities if $g>3$ (see Example 3.2 below for a discussion of the $g=3$ case).

Whenever the Abel-Jacobi map has singular points, the $(1,1)$-form $\mathrm{AJ}_{k}^{*} \Omega_{J}$ obtained as pullback of the polarisation of the Jacobian is degenerate, i.e. its rank drops down. Then one is left 
with a degenerating Kähler metric on $\Sigma^{(k)}$, for which the existence and uniqueness of geodesics associated to any point and direction may not hold. (The corresponding $(1,1)$-form is still closed, as it is the pull-back of the closed 2-form $\Omega_{\mathrm{J}}$ ). We argue in Section 7 of [22], following essentially the same steps of the proof of Theorem 2.3, that $\mathrm{AJ}_{k}^{*} \Omega_{J}$ describes once again the dissolving limit of the $L^{2}$-geometry on the moduli space of $k$-vortices.

In the multivortex case, over the sets of regular points of each $\mathrm{AJ}_{k}$ one thus obtains Kähler metrics that can be regarded as higher-dimensional generalisations of the Bergman metric described above. Effective divisors on the subset where the metrics are regular represent line bundles that do not admit independent holomorphic sections (with different divisors of zeroes). In contrast, in the language of algebraic geometry [1], the induced metric of dissolving vortices is degenerate over special effective divisors, which run or move in nontrivial linear systems. The directions of degeneracy on $\Sigma^{(k)}$ are precisely those along the complete linear system associated with a special divisor $D$. The sets of special divisors $D$, sitting on exceptional fibres of the Abel-Jacobi map, are complex projective spaces whose dimension $\ell$ can be related to sheaf cohomology via the Riemann-Roch theorem [11]:

$$
\begin{aligned}
\ell & =\operatorname{dim}_{\mathbb{C}} \mathbb{P}\left(H^{0}(\Sigma, \mathcal{O}(D))\right) \\
& =\operatorname{dim}_{\mathbb{C}} H^{1}(\Sigma, \mathcal{O}(D))+\operatorname{deg} D-g+1-1 \\
& =\operatorname{dim}_{\mathbb{C}} H^{1}(\Sigma, \mathcal{O}(D))+k-g .
\end{aligned}
$$

The divisor $D$ is special precisely when the following strict inequality holds:

$$
\operatorname{dim}_{\mathbb{C}} H^{1}(\Sigma, \mathcal{O}(D))=\operatorname{dim}_{\mathbb{C}} H^{0}\left(\Sigma, \mathcal{O}\left(K_{\Sigma}-D\right)\right)^{*}>g-k .
$$

The relations among the geometry of linear systems on $\Sigma$, exceptional fibres of the AbelJacobi map, and singularities of the subvarieties $W_{k} \subset \operatorname{Jac}(\Sigma)$ are summarised in the beautiful Riemann-Kempf theorem, which essentially says that a point $w \in W_{k}$ is a singularity of multiplicity $\left(\begin{array}{c}g-k+\ell \\ \ell\end{array}\right)$, its tangent cone being the union of images of the tangent spaces $\mathrm{T}_{D} \Sigma^{(k)}$ by the differential of the Abel-Jacobi map, where the effective divisor $D$ runs over the complete linear system associated with (i.e. is the fibre over) $w$. The subvarieties $W_{k} \subset \operatorname{Jac}(\Sigma)$ are locally given by determinantal equations, and their structure is an important topic in the modern algebraic geometry of curves [1].

In particular, the answer to the natural question of whether $W_{k}(\Sigma) \subset \operatorname{Jac}(\Sigma)$ will happen to be singular or not (i.e. whether special divisors exist) depends on $k$, the genus $g$ of $\Sigma$ and the complex structure on $\Sigma$, and it is part of a rich subject that goes under the name of Brill-Noether theory [1]. A sufficient condition for existence of singularities is given by in following result:

Theorem 3.1. If the inequality $k \geq \frac{g}{2}+1$ is satisfied, then $W_{k}$ is a singular algebraic variety, irrespective of the complex structure of $\Sigma$.

The first proof of this statement was presented by Meis [24] and used complex analysis on Teichmüller spaces, resorting to certain specific models of Riemann surfaces in separate cases of odd and even genus. Subsequently, a number of more conceptual and algebraic proofs were given, some of them generalising Meis's result to linear systems of higher dimension. Kleiman and Laksov constructed a very clean proof [16] that should appeal most to singularity theorists. It makes crucial use of Porteous' formula [27] for the Thom polynomial giving the class of the scheme parametrising special divisors inside the Chow $\operatorname{ring}$ of $\Sigma^{(k)}$, under weaker assumptions [15] than the transversality conditions assumed in the original paper [27].

To illustrate more concretely the behaviour of the Abel-Jacobi map for $k>1$ and the structure of its image $W_{k}$ as a complex $k$-fold inside the Jacobian, we briefly describe examples of the possible behaviours at low vortex number $k$. Typically, the qualitative behaviour at a given genus depends crucially on the complex structure of $\Sigma$, e.g. on whether $\Sigma$ is hyperelliptic, and 
on what kind of linear systems the geometry of $\Sigma$ allows. Needless to say, the situations at higher $k$ and $g$ will be considerably more complicated than these examples. For more information, the reader is referred to the textbooks $[1,25]$.

Example 3.2. For $k=2$, the lowest-genus case where (3.1) is satisfied is $g=3$. Note that these values of $k$ and $g$ do not obey the inequality in Theorem 3.1. In this situation there are two subcases. If $\Sigma$ is a nonhyperelliptic curve (the generic situation), the image $W_{2} \subset \operatorname{Jac}(\Sigma)$ of the Abel-Jacobi map is smooth, and just a copy of the moduli space $\mathcal{M}_{2}=\Sigma^{(2)}$ inside the Jacobian. In fact, this is the only case with $k>1$ where the 2 -form $\mathrm{AJ}_{2}^{*} \Omega_{J}$ is globally nondegenerate on a $\Theta$-divisor, and the dissolving limit metric is regular everywhere. If $g=3$ but $\Sigma$ is hyperelliptic, then $W_{2}$ already has a singularity. $W_{2}$ is the singular complex surface got from the smooth surface $\Sigma^{(2)}$ by blowing down a copy of $\mathbb{C P}^{1}$ to a point, which is a double point in $W_{2}$ [28]. The exceptional $\mathbb{C P}^{1}$ fibre that is blown down is the pencil of degree two divisors that are orbits of the hyperelliptic involution (a $g_{2}^{1}$ ); the space of orbits is the quotient of $\Sigma$ by the hyperelliptic involution, which is a $\mathbb{C P}^{1}$ that embeds in $\Sigma^{(2)}$ holomorphically with noncontractible image. This exceptional fibre has an analogue for any moduli space of 2-vortices on a hyperelliptic curve $\Sigma[5]$.

Example 3.3. If $k=3$, the simplest situation requires $g=4$. Since $3 \geq \frac{4}{2}+1$, Theorem 3.1 guarantees that $W_{3}$ will always contain singularities. In fact, there are three subcases to consider. If $\Sigma$ is not hyperelliptic, one can show that it can be obtained as an intersection of a quadric $Q$ and a cubic $C$ in $\mathbb{C P}^{3}$. The first subcase is when $Q$ is smooth, hence biholomorphic to $\mathbb{C P}^{1} \times \mathbb{C P}^{1}$. Then $C$ meets each projective line of the form $\left\{P_{1}\right\} \times \mathbb{C P}^{1}$ or $\mathbb{C P}^{1} \times\left\{P_{2}\right\}$ in $Q$ at three points, so $\Sigma=Q \cap C$ projects to either of the two $\mathbb{C P}^{1}$ factors of $Q$ as a 3 -cover. The pre-images of points in $\mathbb{C P}^{1}$ by the two projections form effective divisors of degree 3 moving in two pencils (i.e. parametrised by two projective lines), and describe two copies $F_{1}, F_{2}$ of $\mathbb{C P}^{1}$ inside $\Sigma^{(3)}$, which are $g_{3}^{1}$ 's on $\Sigma$. These are the exceptional fibres of the Abel-Jacobi map. The image $W_{3}$ can be obtained by blowing down these rational curves $F_{1}, F_{2}$ to two points, which are ordinary double points of the 3-fold. The second subcase is when $\Sigma$ is not hyperelliptic, hence $\Sigma=Q \cap C$ as before, but now $Q$ is singular (a quadric cone); then $Q$ can be described as a family of projective lines parametrised by a $\mathbb{C P}^{1}$ and all meeting at the singular point. Each line in the family again meets $C$ at three points, and so $\Sigma$ inherits one pencil of degree 3 effective divisors (a $g_{3}^{1}$ ), which is the only exceptional fibre of the Abel-Jacobi map. The image $W_{3}$ in this case is again got by blowing down this $\mathbb{C P}^{1}$ fibre, and this results in a double point in the 3 -fold which has higher multiplicity. The third and last subcase occurs when $\Sigma$ is hyperelliptic. The exceptional fibres here form a complex surface inside $\Sigma^{(3)}$, namely, the locus of effective divisors on $\Sigma$ consisting of adding any point of $\Sigma$ to the $\mathbb{C P}^{1}$ of hyperelliptic orbits described in the previous example; this can be described as a family of pencils (i.e. $g_{3}^{1}$ 's) parametrised by $\Sigma$. Then $W_{3}$ is obtained from $\Sigma^{(3)}$ by blowing down this surface to a curve isomorphic to $\Sigma$.

\section{Dissolving multivortices NeAR a Singularity}

One peculiar aspect of the geometry of multivortices is the degeneration of the underlying Kähler structures at the singularities of the Abel-Jacobi map, as described above, and this will be our focus in the present section. To understand the behaviour of the geodesic flow in a neighbourhood of a singularity, we shall analyse in detail the simplest situation, which occurs in the scattering of two dissolving vortices on a hyperelliptic Riemann surface of genus three.

We start by recalling that the image $W_{2}$ of the Abel-Jacobi map for degree two effective divisors

$$
\mathrm{AJ}_{2}: \Sigma^{(2)} \longrightarrow \operatorname{Jac}(\Sigma)
$$


on a hyperelliptic curve $\Sigma$ with $g=3$ has a double point, whose blow-up is the exceptional fibre in $\Sigma^{(2)}$, which is a projective line (see Example 3.2). This fact essentially goes back to Klein [17]; see e.g. [7] for a modern perspective. Since we are only interested in the leading local behaviour near this critical locus, we will not need to use theta-functions, and will instead take the standard algebraic model

$$
t_{3}^{2}=t_{1} t_{2}
$$

for the double point, using local coordinates $t_{i}: U \rightarrow \mathbb{C}$ centred at the singularity; so (4.2) gives a local equation for the image of $W_{2} \cap U \subset \operatorname{Jac}(\Sigma)$ under the coordinate system, which we may regard as a hypersurface $W_{2}^{\prime}$ in an open neighbourhood $U^{\prime}$ of the origin of $\mathbb{C}^{3}$. Now we blow up $(0,0,0) \in U^{\prime}$, to obtain a 3-fold $\widetilde{U^{\prime}}$ together with a holomorphic map $\pi: \widetilde{U^{\prime}} \rightarrow U^{\prime}$ which has $\pi^{-1}(0,0,0)=\mathbb{P}\left(\mathrm{T}_{(0,0,0)} U^{\prime}\right) \cong \mathbb{C P}^{2}$ but is one-to-one everywhere else. For the benefit of the reader, we recall how this is constructed [2].

The manifold $\widetilde{U^{\prime}}$ can be regarded as the subset of $U^{\prime} \times \mathbb{C P}^{2}$ defined by the incidence relation

$$
t_{i} v_{j}=t_{j} v_{i} \quad \text { for all } \quad i, j \in\{1,2,3\}
$$

where $v_{j}$ are homogeneous coordinates on the projectivisation $\mathbb{C P}^{2}$ of the tangent space at the origin, and the map $\pi$ is simply the projection $\mathrm{pr}_{U^{\prime}}$ onto the first factor. In the open set of $U^{\prime} \times \mathbb{C P}^{2}$ where $v_{3} \neq 0$, for example, $\widetilde{U}^{\prime}$ is described by the system of equations

$$
t_{1}=\frac{v_{1}}{v_{3}} t_{3}, \quad t_{2}=\frac{v_{2}}{v_{3}} t_{3}
$$

which has constant rank 2 , and this determines a 3 -dimensional submanifold. Since the incidence relation (4.3) is trivially satisfied for $\left(t_{1}, t_{2}, t_{3}\right)=(0,0,0)$, we get indeed the whole of the $\mathbb{C P}^{2}$ factor as exceptional fibre.

Imposing the equation (4.2), we obtain a surface $\widetilde{W}_{2}^{\prime} \cap \widetilde{U}^{\prime}$ which is smooth; the singularity is replaced by the conic $v_{3}^{2}=v_{1} v_{2}$ in the exceptional fibre $\mathbb{C P}^{2}$, which is itself a projective line $\mathbb{C P}^{1}$, and the restriction

$$
\left.\pi\right|_{\widetilde{W}_{2}^{\prime} \cap \widetilde{U}^{\prime}}: \widetilde{W}_{2}^{\prime} \cap \widetilde{U}^{\prime} \rightarrow W_{2}^{\prime} \cap U^{\prime}
$$

provides a local resolution of the double point on the surface. To find the resolution map explicitly, we should use a system of two local coordinates where a dense subset of the exceptional fibre is visible; for example, an affine coordinate on the $\mathbb{C P}^{1}$ factor, say $q=\frac{v_{3}}{v_{1}}$, together with one of the coordinates on the first factor, say $p=t_{1}$. In these coordinates, the projection is given by

$$
(p, q) \mapsto\left(t_{1}, t_{2}, t_{3}\right)=\left(p, p q^{2}, p q\right) \in U^{\prime} .
$$

Working on such local patches, it is not hard to see that the projection of $\widetilde{W_{2}^{\prime}} \cap \widetilde{U}^{\prime}$ onto the second factor of $\widetilde{U}^{\prime} \times \mathbb{C P}^{2}$ can be understood as a restriction of the standard projection

$$
\mathrm{T}^{*} \mathbb{C P}^{1} \longrightarrow \mathbb{C P}^{1}
$$

to a neighbourhood of the (image of the) zero section, which gives a very concrete picture of the resolution. The exceptional fibre of $\mathrm{AJ}_{2}$ is identified with the zero section, parametrised by $q$, and our complex coordinate $p$ parametrises the cotangent fibres.

We want to understand the effect of pulling back a Kähler metric on $U^{\prime}$ to the blow-up $\widetilde{U}^{\prime}$, and in particular the behaviour of the geodesic flow near the exceptional fibre where the metric becomes degenerate. The Kähler metric we consider is the standard euclidean metric 
on $U^{\prime}, g_{0}=\left|\mathrm{d} t_{1}\right|^{2}+\left|\mathrm{d} t_{2}\right|^{2}+\left|\mathrm{d} t_{3}\right|^{2}$, as the qualitative behaviour of the flow will not depend on anisotropy factors. Pulling back to $\widetilde{U}^{\prime}$ we obtain

$$
\begin{aligned}
\widetilde{g}=\pi^{*} g_{0}= & \left(1+|q|^{2}+|q|^{4}\right) \mathrm{d} p \mathrm{~d} \bar{p}+|p|^{2}\left(1+4|q|^{2}\right) \mathrm{d} q \mathrm{~d} \bar{q} \\
& +\bar{p} q\left(1+2|q|^{2}\right) \mathrm{d} p \mathrm{~d} \bar{q}+p \bar{q}\left(1+2|q|^{2}\right) \mathrm{d} q \mathrm{~d} \bar{p} .
\end{aligned}
$$

As expected, this tensor defines a Kähler metric in the complement of the complex line with equation $p=0$, but its rank (over $\mathbb{R}$ ) drops from 4 to 2 on this line, which corresponds to an affine piece of the exceptional $\mathbb{C P}^{1}$ fibre of the Abel-Jacobi map. To understand the geodesic flow, we should first compute the Christoffel symbols. For a Kähler metric this calculation simplifies, and moreover Christoffel symbols mixing holomorphic and anti-holomorphic directions automatically vanish [2]. We find:

$$
\begin{aligned}
& \widetilde{\Gamma}_{p q}^{q}=\widetilde{\Gamma}_{q p}^{q}=\frac{1}{p}, \quad \widetilde{\Gamma}_{p q}^{p}=\widetilde{\Gamma}_{q p}^{p}=\widetilde{\Gamma}_{p p}^{q}=\widetilde{\Gamma}_{p p}^{p}=0, \\
& \widetilde{\Gamma}_{q q}^{p}=-\frac{2 p \bar{q}^{2}}{1+4|q|^{2}+|q|^{4}}, \quad \widetilde{\Gamma}_{q q}^{q}=\frac{2 \bar{q}\left(2+|q|^{2}\right)}{1+4|q|^{2}+|q|^{4}} .
\end{aligned}
$$

These lead to the following geodesic equations:

$$
\begin{gathered}
\ddot{p}-\frac{2 p \bar{q}^{2} \dot{q}^{2}}{1+4|q|^{2}+|q|^{4}}=0, \\
\ddot{q}+\frac{2 \dot{p} \dot{q}}{p}+\frac{2 \bar{q}\left(2+|q|^{2}\right) \dot{q}^{2}}{1+4|q|^{2}+|q|^{4}}=0,
\end{gathered}
$$

where the derivatives are with respect to a parameter $s$, say.

An obvious integral of motion is the kinetic energy of the geodesic flow (up to a constant factor),

$$
\left(1+|q|^{2}+|q|^{4}\right)|\dot{p}|^{2}+|p|^{2}\left(1+4|q|^{2}\right)|\dot{q}|^{2}+\left(1+2|q|^{2}\right)(\bar{p} q \dot{p} \dot{\bar{q}}+p \bar{q} \dot{p} \dot{q}),
$$

and there are further integrals of motion arising from the invariance of $\widetilde{g}$ under phase rotations of $p$ and of $q$. The conservation of the kinetic energy already implies that the motion on the exceptional fibre $\mathbb{C P}^{1}$ (parametrised by the coordinate $q$ ) is suppressed in its tangent directions: as $p \rightarrow 0$, all the kinetic energy must be transferred to motion along the transverse directions parametrised by the complex coordinate $p$. In particular, any geodesic intersecting the exceptional fibre must do so at isolated points of the fibre.

To demonstrate that there are indeed geodesics crossing the exceptional fibre, we note that the geodesic equations above are satisfied by the rays of the tangent cone to $W_{2}^{\prime}$, i.e. paths of the form $s \mapsto(p, q)=\left(c_{1} s, c_{2}\right)$ for constants $c_{1} \in \mathbb{C}^{*}$ and $c_{2} \in \mathbb{C}$. These correspond to lifts of real straight lines on $U^{\prime}$ towards the singularity, which hit a point on the exceptional fibre corresponding to the complex tangent direction their velocity represents, and then continue along the same real direction. Since the exceptional fibre is reached in finite time, the metric on the complement of the exceptional fibre in $\widetilde{W_{2}}$ is not complete.

In fact, such straight ray geodesics are the only geodesics reaching the exceptional fibre $\mathbb{C P}^{1}$. To see this, note first that, as long as $\dot{p}$ is not constant, (4.11) implies that $\dot{q}$ cannot be zero. Dividing equation (4.12) by $\dot{q}$ (assumed to be nonzero) and extracting the real part of the resulting equation, we obtain a new differential equation,

$$
\frac{\ddot{q}}{\dot{q}}+\frac{\ddot{\bar{q}}}{\dot{\bar{q}}}+\frac{2 \dot{p}}{p}+\frac{2 \dot{\bar{p}}}{\bar{p}}+\frac{2\left(2+|q|^{2}\right)(\bar{q} \dot{q}+q \dot{\bar{q}})}{1+4|q|^{2}+|q|^{4}}=0
$$


which can be integrated to conclude that

$$
\left(1+4|q|^{2}+|q|^{4}\right)|p|^{4}|\dot{q}|^{2}
$$

is another integral of motion. Thus for $p$ to reach zero, $\dot{q}$ would have to blow up, which cannot happen. Initial conditions that try to reach the exceptional fibre with initial velocities having nontrivial tangent component along the $\mathbb{C P}^{1}$ will be forced to flow rapidly around this 2 -sphere as they approach it transversely.

In terms of vortex motion, the effect of the singularity is that motion along the special linear system is suppressed. So whenever two vortices reach points on the surface that are related by the hyperelliptic involution, they will be unable to move to neighbouring pairs of points that are also related by the involution. In particular, in the dissolving limit it will be impossible to make vortices collide head-on onto a Weierstraß point of the surface: these are precisely the branch points of the two-fold holomorphic branched cover $\sigma: \Sigma \rightarrow \mathbb{C P}^{1}$, and geodesics through them are tangentially preserved by the hyperelliptic involution near the branch point. More precisely, we know from the discussion above that the only geodesics through the $\mathbb{C P}^{1}$ with equation $p=0$ must cross with $\dot{q}=0$, whereas we have:

Proposition 4.1. A frontal collision of two vortices at a fixed point $W \in \Sigma$ of the hyperelliptic involution occurs at right angles and with $\dot{q} \neq 0$.

Proof. Let $z \in \mathcal{O}_{\Sigma, W}$ denote a local parameter in $\Sigma$ at the point $W$, a generator of the maximal ideal $\mathfrak{n}_{\Sigma, W}$ in the local ring [20]. We have been using $q$ to denote any coordinate on the exceptional fibre $\mathbb{C P}^{1}$ of the Abel-Jacobi map, and now we shall also assume without loss of generality that its image in the local ring $\mathcal{O}_{\mathbb{C P}^{1}, \sigma(W)}$ is a local parameter. Since the map $\sigma$ has ramification index two at $W$, one has $\sigma^{*} q=u z^{2}$ for some unit $u \in \mathcal{O}_{\Sigma, W}^{\times}$.

We denote by $\Delta$ the natural embedding via the diagonal inclusion

$$
\Delta: \Sigma \hookrightarrow \Sigma \times \Sigma \stackrel{\tilde{\pi}}{\longrightarrow} \Sigma^{(2)}=\Sigma^{2} / \mathfrak{S}_{2} .
$$

Note that $z$ induces local parameters $z_{1}, z_{2}$ in $\Sigma^{2}$ at $(W, W)$ in the obvious way, and from them one obtains a system of local parameters $s_{1}, s_{2}$ on $\Sigma^{(2)}$ at $\Delta(W)$ via the fundamental theorem on symmetric functions, i.e. the map of local rings induced by $\tilde{\pi}$ relates $\tilde{\pi}^{*}\left(s_{1}\right)=z_{1}+z_{2}, \tilde{\pi}^{*}\left(s_{2}\right)=$ $z_{1} z_{2}$. The image $\Delta(\Sigma)$ is described by the equation $s_{1}^{2}-4 s_{2}=0$ locally at $\Delta(W)$ in $\Sigma^{(2)}$, and we can compute

$$
\begin{aligned}
\mathrm{T}_{\Delta(W)}^{*} \Delta(\Sigma) & =\mathfrak{n}_{\Sigma^{(2)}, \Delta(W)} /\left(\mathfrak{n}_{\Sigma^{(2)}, \Delta(W)}^{2}+\left(s_{1}^{2}-4 s_{2}\right)\right) \\
& =\left(s_{1}, s_{2}\right) /\left(s_{1}^{2}, s_{2}^{2}, s_{1} s_{2}, s_{1}^{2}-4 s_{2}\right) \\
& =\left(s_{1}\right) /\left(s_{1}^{2}\right) .
\end{aligned}
$$

Let $\iota: \mathbb{C P}^{1} \hookrightarrow \Sigma^{(2)}$ denote the inclusion of the $g_{2}^{1}$. It induces a surjective map of local rings $\iota^{*}: \mathcal{O}_{\Sigma^{(2)}, \Delta(W)} \rightarrow \mathcal{O}_{\mathbb{C P}^{1}, \sigma(W)}$. Since the intersection of the images $\Delta(\Sigma)$ and $\iota\left(\mathbb{C P}^{1}\right)$ is transverse at $\Delta(W) \in \Sigma^{(2)}$, the calculation above implies that $\iota^{*} s_{2}$ must be a local parameter; so there is also a unit $v \in \mathcal{O}_{\mathbb{C P}^{1}, \sigma(W)}^{\times}$with $\iota^{*} s_{2}=v q$. Hence we obtain in $\mathcal{O}_{\Sigma, W}$

$$
z^{2}=\tilde{u}(\iota \circ \sigma)^{*} s_{2}
$$

with $\tilde{u}=u \sigma^{*} v \in \mathcal{O}_{\Sigma, W}^{\times}$.

A collision of two vortices at $W \in \Sigma$ can be described by a parametrisation $t \mapsto\left(z_{1}(t), z_{2}(t)\right)$ with $t \in(-\epsilon, \epsilon), \epsilon>0$ and $z_{1}(0)=z_{2}(0)=0$; the collision is frontal if moreover $\dot{z}_{1}(0)=$ $-\dot{z}_{2}(0)$, which implies $\dot{s}_{1}(0)=0$. Then necessarily $\dot{s}_{2}(0) \neq 0$. From equation (4.16) we obtain infinitesimally close positions of the vortices by taking square roots, which justifies the assertion on the scattering at right angles. (We note in passing that scattering at right angles is a 
well-known feature of the frontal scattering of vortices for regular $L^{2}$-metrics on their moduli spaces [12].) Finally, we obtain in the local ring at $\sigma(W)$ (or the pull-back to $\Sigma^{(2)}$ )

$$
\dot{q}(0)=\dot{v}(0) s_{2}(0)+v(0) \dot{s}_{2}(0)=v(0) \dot{s}_{2}(0) \neq 0 .
$$

\section{REFERENCES}

[1] E. Arbarello, M. Cornalba, P.A. Griffiths and J. Harris: Geometry of Algebraic Curves, vol. 1, Springer, 1985

[2] W. Ballmann: Lectures on Kähler Manifolds, European Mathematical Society, 2006

[3] J.M. Baptista and N.S. Manton: The dynamics of vortices on $S^{2}$ near the Bradlow limit. J. Math. Phys. 44 (2003) 3495-3508 DOI: 10.1063/1.1584526

[4] E.B. Bogomolny: The stability of classical solutions. Sov. J. Nucl. Phys. 24 (1976) 449-454

[5] M. Bökstedt and N.M. Romão: On the curvature of vortex moduli spaces. arXiv:1010.1488

[6] S.B. Bradlow: Vortices in holomorphic line bundles over closed Kähler manifolds. Commun. Math. Phys. 135 (1990) 1-17 DOI: 10.1007/BF02097654

[7] V.M. Buchstaber, V.Z. Enolskil and D.V. Leykin: Kleinian functions, hyperelliptic Jacobians and applications. Rev. Math. Math. Phys. 10 (1997) 1-125

[8] S. Donaldson and P. Kronheimer: The Geometry of Four-Manifolds, Clarendon Press, 1990

[9] G. Dunne: Self-Dual Chern-Simons Theories, Springer, 1995 DOI: 10.1007/978-3-540-44777-1

[10] Ó. García-Prada: A direct existence proof for the vortex equations over a compact Riemann surface. Bull. London Math. Soc. 26 (1994) 88-96 DOI: 10.1112/blms $/ 26.1 .88$

[11] P. Griffiths and J. Harris: Principles of Algebraic Geometry, Wiley, 1978

[12] N.J. Hitchin: The geometry and topology of moduli spaces; in: M. Francaviglia and F. GherARdelli (Eds.): Global Geometry and Mathematical Physics (Montecatini Terme 1988), Springer, 1990 DOI: $10.1007 / \mathrm{BFb} 0085064$

[13] A. JAFfe and C. TAubes: Vortices and Monopoles, Birkhäuser, 1980

[14] J. Jost: Compact Riemann Surfaces, 3rd edition, Springer, 2006 DOI: 10.1007/978-3-540-33067-7

[15] G. Kempf and D. Laksov: The determinantal formula of Schubert calculus, Acta. Math. 132 (1973) 153-162 DOI: 10.1007/BF02392111

[16] S.L. Kleiman and D. Laksov: Another proof of the existence of special divisors, Acta Math. 132 (1974) 163-176 DOI: $10.1007 /$ BF02392112

[17] F. KLein: Über elliptische Sigmafunktionen, Math. Ann. 27 (1886) 341-464

[18] Sh. Kobayashi: Geometry of bounded domains, Trans. Math. Am. Soc. 92 (1959) 267-290 DOI: 10.1090/S0002-9947-1959-0112162-5

[19] J. Lewittes: Differentials and matrices on Riemann surfaces, Trans. Am. Math. Soc. 139 (1969) 311-318

[20] S. Łojasiewicz: Introduction to Complex Analytic Geometry, Birkhäuser, 1991

[21] N.S. Manton and S.M. Nasir: Volume of vortex moduli spaces, Commun. Math. Phys. 199 (1999) 591-604 DOI: $10.1007 / \mathrm{s} 002200050513$

[22] N.S. Manton and N.M. Romão: Vortices and Jacobian varieties, J. Geom. Phys. 61 (2011) 1135-1155 DOI: $10.1016 /$ j.geomphys.2011.02.017

[23] N. Manton and P. Sutcliffe: Topological Solitons, Cambridge University Press, 2004 DOI: 10.1017/CBO9780511617034

[24] T. Meis: Die minimale Blätterzahl der Konkretisierungen einer kompakten Riemannschen Fläche, Schr. Math. Inst. Univ. Münster 16 (1960) 1-61

[25] D. Mumford: The Red Book of Varieties and Schemes, 2nd edition, Springer, 2004

[26] S.M. NAsir: Vortices and flat connections, Phys. Lett. B 419 (1990) 253-257 DOI: 10.1016/S03702693(97)01465-2

[27] I.R. Porteous: Simple singularities of maps; in: C.T.C. WAll (Ed.): Proceedings of Liverpool Singularities Symposium I (Liverpool 1969/70), Springer, 1971

[28] M. Reid: Chapters on Algebraic Surfaces; in: J. Kollár (Ed.): Complex Algebraic Geometry (Park City UT 1993), American Mathematical Society, 1997; alg-geom/9602006

[29] T.M. Samols: Vortex scattering, Commun. Math. Phys. 145 (1992) 149-179 DOI: 10.1007/BF02099284

[30] I.A.B. Strachan: Low-velocity scattering of vortices in a modified Abelian Higgs model. J. Math. Phys. 33 (1992) 102-110 DOI: 10.1063/1.529949

[31] D. Stuart: Dynamics of Abelian Higgs vortices in the near Bogomolny regime, Commun. Math. Phys. 159 (1994) 51-91 DOI: 10.1007/BF02100485 
[32] D.M.A. Stunar: Periodic solutions of the abelian Higgs model and rigid rotation of vortices, Geom. Funct. Anal. 9 (1999) 568-595 DOI: 10.1007/s000390050096

[33] Y.-S. YANG: Solitons in Field Theory and Nonlinear Analysis, Springer, 2001

Departament D’Àlgebra i Geometria, Universitat de Barcelona, Gran Via de les Corts CataLanes 585, 08007 Barcelona, Spain

Max-Planck-Institut für Mathematik, Vivatsgasse 7, 53111 Bonn, Germany

E-mail address: nromao@imf.au.dk 